Scottish Universities Insight Institute (SUII): ACCELERATING PROGRESS TOWARDS THE UN SUSTAINABLE DEVELOPMENT GOALS

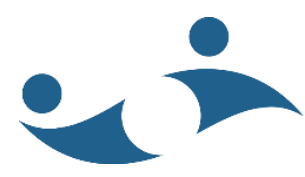

\title{
Driving the transition to a resilient and inclusive future: the role of the ocean
}

\author{
Second Workshop Report
}

\section{Mobilising the science community for a sustainable and inclusive ocean economy}

July 2021

Project partners

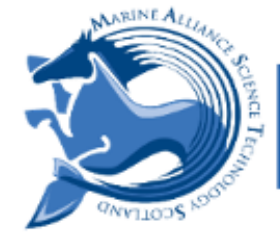

\section{HERIOT}

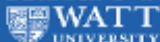
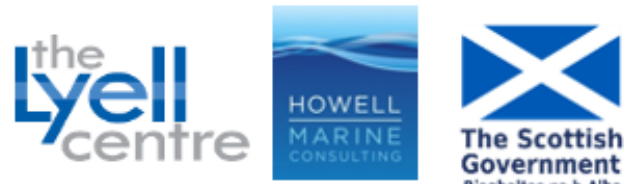

marinescotland

Government

science
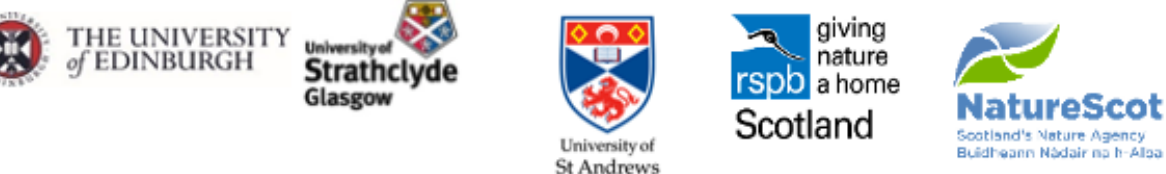

UNIVERSITY of

STIRLING

Programme Team: Dr Chris Leakey (University of St Andrews / MASTS), Dr Daniela Diz (Heriot Watt University), Dr Lucy Greenhill (Howell Marine Consulting), Dr Estelle Jones (Scottish Government), Prof. Murray Roberts (University of Edinburgh), Dr Ingrid Kelling (Heriot Watt University), Dr Clive Mitchell (NatureScot), Prof. Mike Heath (University of Strathclyde), Prof. Trevor Telfor (University of Stirling), Prof. Selina Stead (University of Stirling), Alex Kinninmonth (RSPB Scotland), Prof. Andrew Brierley (University of St Andrews), Dr Tim Stojanovic (University of St Andrews) and Dr Barbara Berx (Marine Scotland Science). 


\section{Table of Contents}

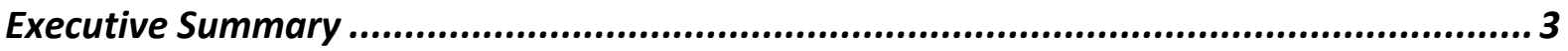

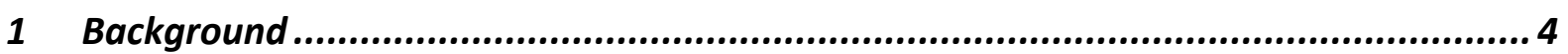

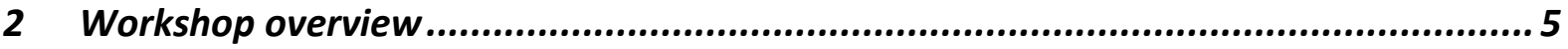

3 The role of science in supporting the Blue Economy.............................................6

What does science contribute to Blue Economy policy development and implementation?........ 6

What are the challenges in integrating science into policy and decision-making for the Blue

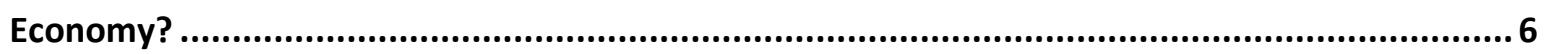

How can we better engage scientists in the Blue Economy? ......................................................

4 Science for managing synergies and trade-offs............................................. 8

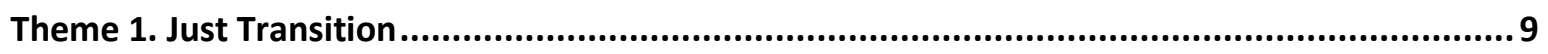

What research and knowledge can help us manage synergies and trade-offs in a Just Transition?............9

Where is innovation needed to promote synergies in a Just Transition? ............................................... 9

What type of indicators, data and evidence are needed to measure progress towards a Just Transition? 10

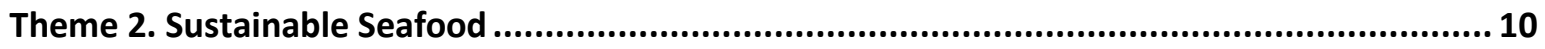

What research and knowledge can help us manage synergies and trade-offs in sustainable seafood? .....10

Where is innovation needed to promote synergies? ............................................................................... 11

What type of indicators, data and evidence are needed to measure progress towards sustainable seafood

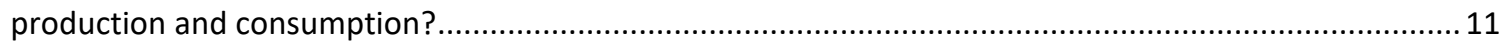

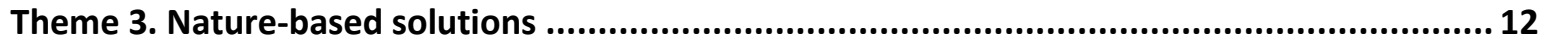

What research and knowledge can help us manage synergies and trade-offs in nature-based solutions? 12 Where is innovation needed to promote synergies? .......................................................................... 12

What type of indicators, data and evidence are needed to measure progress? ..................................... 12

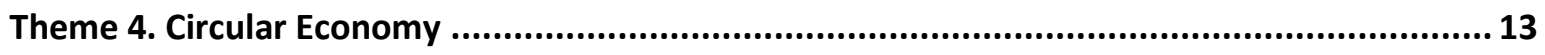

What research and knowledge can help us manage synergies and trade-offs for a circular economy? .....13

Where is innovation needed to promote synergies? ........................................................................ 13

What type of indicators, data and evidence are needed to measure progress? .................................... 13

Suggested citation: Greenhill, L., Leakey C. and Diz, D. 2021. Mobilising the science community in progressing towards a sustainable and inclusive ocean economy. Workshop Report (15 ${ }^{\text {th }}$ June 2021). Scottish Universities Insights Institute, UK. 


\section{Executive Summary}

Through a programme of activities from January to July 2021, this Scottish Universities Insight Institute (SUII) project focussed on accelerating progress towards the 'Just Transition' for an environmentally sustainable, resilient and equitable economy and society in Scotland, within the framework provided by the UN Sustainable Development Goals (SDGs). Through a series of activities to support dialogue between the scientific and policy communities in Scotland, the programme aimed to:

1. Develop our understanding of interdependencies between marine and cross-cutting policy themes to promote policy coherence, promoting synergies and managing trade-offs.

2. Mobilise the science and policy communities in co-developing knowledge for policy impact, including understanding data and evidence needs for innovation and measuring progress.

Our first event was held on $27 \& 28^{\text {th }}$ January 2021 and focussed on understanding and developing policy coherence in Scotland. Through the nexus themes of 'Climate' and 'Seafood', connections were identified across a breadth of policy themes, along with key actions and opportunities to promote synergies and manage trade-offs. This report summarises our second virtual event held on the $15^{\text {th }}$ June 2021, which advanced the Event 1 discussions to focus specifically on the role of science in informing policy in addressing a sustainable Blue Economy.

Across the Blue Economy, science must play a fundamental role in moving us away from business as usual to a more sustainable pathway. It provides evidence to inform policy by understanding baselines, trends and tipping points, as well as the multiple and interacting effects of human activities and policy interventions. Measuring progress depends on strong evidence and requires the design of a monitoring framework based on well-defined objectives and indicators, informed by the diverse disciplines required to inform progress on cross-cutting policy objectives such as the Just Transition.

The differences between the scientific and policy processes are stark and affect interaction between them, including, among other factors, the time pressures of governmental decision-making, and the lack of support and reward in academia for policy engagement. To enable improved integration, the diverse nature of the science / policy interface is important to recognise - improved communication between scientists and policy professionals within government is important, as well as interaction with the wider academic community through secondments and other mechanisms. Skills in working across boundaries are valuable, requiring training and professional recognition.

We also discussed the science needs across the themes of the Just Transition, Sustainable Seafood, Nature-based Solutions and the Circular Economy, where we considered:

- What research and knowledge can help us manage synergies and trade-offs?

- Where is innovation needed to promote synergies?

- What type of indicators, data and evidence are needed to measure progress?

The insights developed through dialogue among participants on these themes are outlined in Section 4 of this report. All project outputs are published on the project webpage. 


\section{Background}

Through a programme of activities from January to July 2021, this Scottish Universities Insight Institute

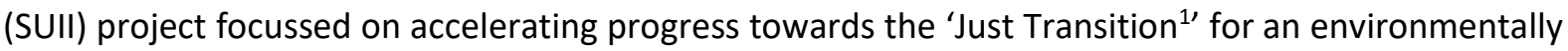
sustainable, resilient and equitable economy and society in Scotland, within the framework provided by the UN Sustainable Development Goals (SDGs). Through a series of activities to support dialogue between the scientific and policy communities in Scotland, the six-month programme aimed to:

1. Develop our understanding of interdependencies between marine and cross-cutting policy themes to promote policy coherence, promoting synergies and managing trade-offs.

2. Mobilise the science and policy communities in co-developing knowledge for policy impact, including understanding data and evidence needs for innovation and measuring progress.

The SUII project was led by Associate Professor Daniela Diz (Heriot-Watt University / Lyell Centre), Dr Chris Leakey (St Andrews University) and Dr Lucy Greenhill (Howell Marine Consulting), supported by a Project Team from Marine Scotland Science, Scottish Government (International Development), NatureScot, the Universities of Edinburgh, Strathclyde, St Andrews and Stirling, Heriot Watt University, and RSPB Scotland.

Two workshops were held from January to July 2021. The report of Event $1^{2}$ summarises the virtual events held on the $27^{\text {th }}$ and $28^{\text {th }}$ January 2021, at which we examined connections across diverse policy areas, looking beyond 'marine' policy to cross-cutting policy themes, connecting different government departments and engaging scientists from across disciplines. Through the nexus themes of 'Climate' and 'Seafood', connections were identified across a breadth of policy themes, along with key actions and opportunities to support policy coherence, promote synergies and manage trade-offs. The Event 1 report provides useful background to this second report.

At Event 2, held on the $15^{\text {th }}$ June 2021, we advanced the Event 1 discussions to focus more specifically on the role that science plays in supporting and enabling a coherent and effective approach to the Blue Economy. We maintained focus on four recurring topics - the just transition, nature-based solutions, the circular economy and sustainable seafood - to consider how science and policy can better interact in understanding synergies and trade-offs, how these might be managed, and how to measure progress towards cross-cutting policy objectives. We also considered the development of the Blue Economy Action Plan (BEAP) in Scotland and identified key actions for the science and policy communities to support its development and implementation.

The workshops were attended by a wide range of academics from across Scottish Universities, Scottish Government including Marine Scotland Science, as well as policy professionals from numerous

\footnotetext{
1 "The imperative of a just transition is that Governments design policies in a way that ensures the benefits of climate change action are shared widely, while the costs do not unfairly burden those least able to pay, or whose livelihoods are directly or indirectly at risk as the economy shifts and changes." Just Transition Commission: A National Mission for a fairer, greener Scotland: https://www.gov.scot/publications/transition-commissionnational-mission-fairer-greener-scotland/

${ }^{2}$ Greenhill, L., Leakey C. and Diz, D. 2021. Driving the transition to a resilient and inclusive future: the role of the ocean and policy coherence. Workshop Report (27th and 28th January 2021). Scottish Universities Insights Institute, UK. DOI: doi.org/10.15664/10023.23456
} 
government departments and public sector organisations, such as Crown Estate Scotland, NatureScot and Highlands and Islands Enterprise. Fisheries Innovation Scotland and some environmental NGOs were also represented.

\section{Workshop overview}

Following reflections and key outcomes from Event 1 presented by Dr Lucy Greenhill of Howell Marine Consulting, we welcomed contribution from our keynote speakers, Professor Colin Moffat, Visiting Professor, Robert Gordon University and former Chief Scientific Advisor Marine to Scottish Government, followed by Amy McQueen, Head of Scottish Government's Marine Analytical Unit (MAU) and Senior Economist.

Colin reflected on his time in post as Chief Scientific Advisor and a vision for the interaction between marine science and policy to drive progress on SDG delivery in the context of the UN Decade of Ocean Science for Sustainable Development. Key points included:

- The magnitude and urgency of the challenges facing us, and which require a 'revolution' not 'evolution' in science for policy.

- The framing of the UN Decade as "The Science we Need for the Ocean We Want" as maintaining the sense we are in control and focussed on benefits for humanity rather than the long-term needs of nature.

- The role of science in understanding the direction of travel, including Scotland's Marine Assessment ${ }^{3}$ and its role in informing future policy direction.

- The importance of the social sciences which are coming to the fore in ocean research and policy, as well as indigenous knowledge, in addition to the traditional science of observations and determining thresholds.

- Accessible and relatable language is key, in policy as well as public engagement more broadly.

- Collaboration across sectors, disciplines and policy areas is essential, which is already done well in Scotland, supported by organisations such as MASTS, but we can go further in delivering the transformative science needed.

- Scientists need to be able to share their knowledge with the people that need to know it, to turn science into evidence we can use.

- Politics requires consensus (or compromises) which generally means the solutions will not be perfect. Scientists need to accept this and help policy find the acceptable balance.

Amy McQueen outlined the remit, structure and function of the Marine Analytical Unit (MAU) at Scottish Government and how this supporting the development of the new Blue Economy Action Plan (BEAP) for Scotland. Key messages were:

- Science helps set policy direction, monitor/evaluate progress and fill key evidence gaps.

- MAU have their own scientists and data experts but are also looking for the best ways to engage with the science community, including natural and social scientists.

- Early collaboration is particularly helpful in identifying and steering research to address priority evidence gaps, to overcome what is often a timing mismatch between policy and science.

- The BEAP (and its science inputs) will help drive policy to tackle synergies and trade-offs.

\footnotetext{
${ }^{3}$ https://marine.gov.scot/sma/
} 
- Logic modelling is being developed to inform the BEAP, to explore the relationship between policy activities and interventions and short, medium and long-term outcomes, and is an important engagement tool for working across departments and stakeholders.

- A suite of baseline indicators exist but MAU are aware that others (including academics) likely have other data which could make a valuable contribution.

- Evidence and data to support social and cultural outcomes is less mature and/or not necessarily available at sufficiently local scale, and there is scope for much more development of work with the social sciences.

These keynote talks were followed by facilitated breakout discussions across five parallel groups which are summarised in sections 3 and 4 , below.

\section{The role of science in supporting the Blue Economy}

\section{What does science contribute to Blue Economy policy development and implementation?}

Science is fundamental to the Blue Economy, as a 'knowledge-based' economy which should move us away from business as usual to a more sustainable pathway. It is critical in providing evidence to inform policy and management and justifying policy positions, including realistic targets for sustainable growth, as well as evaluating whether policy is working. Scientists have a capacity to innovate, to think 'outside the box' and to take an objective (apolitical) long-term and systemsthinking approach that can transcend some of the political and cultural challenges. However, it is also important to be honest about the limitations of science in informing decision-making by policy makers.

The fundamental evidence base must consider the footprints of activities, pressures, effects and management responses, including long term trends and datasets. Measuring progress requires a strong baseline understanding to support long-term monitoring and development of management measures. Indicators for the BEAP must be of sufficiently high resolution to inform progress and be SMART to assess whether management is successful. They must also be relevant to the type of monitoring, e.g. ecosystem condition, sector compliance, operational impacts and environmental surveillance. Where natural capital indices and approaches are proposed, these must be sciencebased, informed by robust valuation and with integration of natural and social sciences, including economics.

We should be co-ordinating on research and technologies across sectors, for example reducing marine industry emissions, and there is a need to support closer collaborative working between 'competing' sectors in the Blue Economy.

What are the challenges in integrating science into policy and decision-making for the Blue Economy?

Time and money are the main challenges faced by both scientists and policy professionals, with research and government departments facing significant funding challenges and competition for resources, and policy makers often have limited time to invest in engagement with research. There is also a time lag between science and policy, with a mismatch between the scientific process and the pressure for delivery of key messages for policy in a timely way, and changes to policy priorities can happen quickly in response to changing politics or crises, for example. This can be problematic as long 
time series of data are needed to understand change, but it is difficult to secure stable funding beyond specific research projects.

There is a challenge in finding a balance between the complexity of the system which science tries to understand and which policy makers try to simplify. A balance is needed so that policy makers can make decisions despite the uncertainty, but over-simplification of the complexities is problematic, particularly in the marine area.

Ability to work across the science / policy boundary is critical, but there is a lack of common language and a shortage of those skilled as knowledge brokers, operating across and between science and policy. The way science is funded, including the Research Excellence Framework (REF), has a distorting impact on how the Higher Education sector operates by undervaluing social capital, networks and public engagement compared to empirical evidence. Further, we need to support generalist skills as a valid and valuable professional direction within academia, rather than focussing solely on rewarding specialisms. Interdisciplinarity is an important precursor to policy integration. Some translation of science and communication of policy interventions to stakeholders is enabled by interaction between Marine Scotland Science and Marine Scotland policy teams, and with public agencies and other authorities. This can be further enhanced and there are benefits from scientists developing a more direct experience of policy development and implementation.

For accessing scientists and the research community, more could be done to promote government open tender opportunities to the science community, potentially helping sourcing and development of different types of expertise. There can be a 'rut' of using existing research contacts rather than new evidence providers and the right networks are not necessarily easy to identify or allocate time to.

Developing science to support policy also requires ensuring that research funding councils and their grant design and application evaluation procedures include policy and science representatives.

How can we better engage scientists in the Blue Economy?

Developing an agreed goal or vision across science and policy is needed to focus effort, find the right balance between long and short-term priorities and develop a shared understanding of the timescales for providing evidence/data. This requires taking the time to 'horizon scan' to identify science priorities for policy, ensuring transparent, robust and independent conclusions.

Focussing on common objectives and use of common language is needed, and we must be more aware of terminology and explanations that help understanding and transparency across disciplines and sectors. Training of policy staff on science including seminars and workshops, and vice versa, can support finding common ground and support collaborative ideas while building knowledge, trust and networks. Events such as these are noted as valuable in supporting dialogue, allowing policy colleagues to speak freely, learn and develop ideas without being constrained and expected to provide the 'policy position'.

As identified in Event 1, bringing scientists into government and agencies, and vice-versa (e.g. increasing secondments) would be valuable, noting that a 'scientist' is not a homogenous concept as they are represented across different organisations, including the public sector, private sector and NGOs.

Independent and critical panels can be useful in providing advice and developing ideas, noting the challenge of multiple groups addressing specific topics within the system. There is a need to further 
explore useful techniques and mechanisms to co-create a shared evidence base and focus on solutions. For example, we could consider forming focussed science advisory groups to support government on developing specific sets of indicators and data streams for the BEAP.

A transparent framework for reporting on how evidence has informed advice, with explanations for alternative policy decisions where deviating from advice would increase accountability, support motivation and improve understanding of the policy process among scientists.

Academic culture can be constraining as policy engagement is not valued and rewarded, and this is a long-standing issue. This needs to change so that networks, outreach and social capacity are properly valued, so policy-science connections are established and supported. We need to value person-toperson connections and knowledge networks. Researchers need to be enabled to engage meaningfully with both national and regional policy processes and there is an opportunity to be more assertive in connecting the marine science community to activities within parliament (e.g. SPICe and SPAN).

We should also consider the role of scientists in informing society for the transformational change that is required to achieve sustainable development. Given the urgency of the challenges faced, we may need better mechanisms for fast tracking science into governance and decision making, and scientists should be prepared to provide advice, clarifying uncertainty and options.

Knowledge brokers and boundary organisations are critical and we could consider a stronger role and resourcing for agencies and knowledge brokers between science and policy, including MASTS and other organisations, including consultancies, in developing science / policy interactions and outputs including policy briefs.

\section{Science for managing synergies and trade-offs}

In this session we used three questions to guide our discussion on the role of science in addressing synergies and trade-offs across the four themes: the Just Transition, Sustainable Seafood, NatureBased Solutions and the Circular Economy.

Across all, it is evident that better links between natural and social sciences are essential in understanding synergies and trade-offs. This includes development of socio-ecological models which can support understanding the links between ecosystem functioning, ecosystem services and societal goods and benefits, as well as cumulative impacts in order to understand costs, benefits and manage competing policy issues.

Innovation is needed in multiple areas across the themes, including:

- Education, including supporting early career researchers (ECRs) and mid-career professionals in thinking in system terms and working across disciplines.

- Innovation needed in communication and stakeholder engagement, particularly for justifying policy positions and difficult choices in the Blue Economy. This capacity to manage difficult conversations requires time, skill and a safe space for inclusive discussions.

- Engagement techniques across 'siloes' and people working in different topics or disciplines within government and externally.

- Development and testing of new approaches to governance.

In measuring progress, there needs to be a co-ordinated approach to developing and managing evidence and linking this to management decisions. 
What research and knowledge can help us manage synergies and trade-offs in a Just Transition?

The Just Transition centres on the implications of the energy transition on society at different scales, with relevance across society as we pursue the shift to 'net zero' and other transformative changes within sectors required to address sustainability. A lack of clarity in the concept was noted, and developing a shared understanding of what is meant by a 'just transition' across stakeholders is necessary, with clear challenges in reaching a common definition of the concept in practice.

Within this theme, social science is fundamental in evaluating and ensuring an inclusive and fair approach, with implications for society central to the concept. This includes ensuring inclusivity in engagement activities and fair representation of views to inform decision-making. Specific requirements include understanding distribution of economic benefits and how these are shared, as well as the barriers in accessing new opportunities. Innovative approaches are needed to understand attitudes and perspectives and evaluate social implications, as well as understand behaviour change and the potential to adapt during the transition. We also need to consider and test new models of ownership and management to support distribution of benefits locally. Social Impact Assessment Guidance is being developed by Marine Scotland and will be important in addressing the implications of development in terms of a Just Transition.

Related to other topics, science is also needed to explore potential future energy scenarios, including spatial distribution of pressures to understand interactions and coexistence and even mutual benefits in the context of a Just Transition. Our understanding of acceptable magnitude and scale of change and its socio-economic implications (i.e. what is 'just'?) must be based on our understanding of environmental limits and tipping points, in relation to climate change as well as the potential impacts of renewable energy development. Definition of the 'just transition' across scales and regions is needed, for example the risks of sea level rise to low lying island areas, to enable a coherent approach. Island communities and fishing communities will be more significantly impacted, as well as the city of Aberdeen with the decline in the oil and gas industry.

Everyone has to be brought together in achieving an ambitious goal for net zero in Scotland, which in turn can serve as an example for other areas globally. At the national level, there are opportunities in Scotland to address the Just Transition including investing in renewables.

In supporting the energy transition, clarity is needed on how support is provided and justified, including incentives to move away from unsustainable practices. Caution is needed where there may be negative impacts of decarbonisation, such as the link between the rapid growth of green technologies, including batteries, and the growing demand for deep sea mining for necessary materials. A transparent and well monitored approach is needed.

\section{Where is innovation needed to promote synergies in a Just Transition?}

- Digital tools to modernise and grow stakeholder engagement, including inclusion of younger people.

- For the identification of and access to disparate data sources, including the informal, commercially sensitive, local and qualitative sources.

- New skills and adapting to new energy systems and is also relevant to a broader post-Covid context. 
- Broader/different skill sets (e.g. health, energy, education, etc.) need to be engaged alongside and integrated with marine science.

- Learning from other non-marine experiences, including terrestrial examples such as coal industry transition, agro-ecology practices replacing industrial agriculture.

- Community-ownership models to promote local benefits, linked to new advances in decentralised governance.

- Grid and transmission solutions to diversify and devolve energy transmission and associated benefits.

- Integration of the rights of future generations in planning and management

- Co-location of different sectors (e.g. energy and aquaculture) requires technological but also new approaches to governance and collaborative business development.

- Innovation in integrated management is key for identifying synergies and addressing tradeoffs, while noting that there is always a need for compromise.

- Modelling frameworks that incorporate social aspects are important but are difficult to integrate in practice.

What type of indicators, data and evidence are needed to measure progress towards a Just Transition?

- Historical data is relevant and we should look at what have we learned from previous transitions, including the oil and gas energy transition in Scotland / UK.

- In measuring progress, we need to collect data beyond Local Authority level to understand community scale implications. Local knowledge/data and lived experience (qualitative data) will be important.

- Longitudinal data is needed to understand community-level transitions, potentially through national census.

- Socio-economic analyses must be rebalanced to give fuller attention to 'socio' aspects including well-being outcomes.

- Seek indicators that stretch across economy, social and nature aspects. Does a 'natural capital' approach lend itself to this?

- We need to understand ecosystem-level responses under different energy scenarios (across all North Sea countries, not only Scotland) and what this means for environmental, social and economic outcomes at various scales.

- Value chain analysis coupled with social analysis to inform indicators of deprivation, poverty and inequality has been deployed successfully in some small island developing areas, and could offer solutions in assessing and addressing trade-offs. This coupling of value chain analysis with social and environmental indicators of offshore wind, for instance, can help address the trade-offs.

\section{Theme 2. Sustainable Seafood}

What research and knowledge can help us manage synergies and trade-offs in sustainable seafood?

There are clear research needs under this theme including the contribution of fishing to climate change (i.e. emissions from vessels and blue carbon habitats' disturbance) and how we can reduce associated impacts. Research is needed to further identify and understand ecologically important areas in Scottish waters (e.g. spawning and nursery grounds, blue carbon habitats), as well as assess the effectiveness of current fisheries management measures for protecting seafloor integrity. 
Therefore, the involvement of scientists and of industry in developing solutions to associated challenges is strongly encouraged. The links between nature-based solutions and fisheries is therefore a strong one and the points addressed in the nature-based solutions session are also relevant to the fisheries context. Understanding the effects of climate change on fish stocks (e.g. migration patterns, etc) is also needed, including with respect to identifying new opportunities and their management needs for sustainable exploitation.

In addition to the need for sustainability in marine capture fisheries, it is also important to ensure sustainable aquaculture practices. This includes considerations on chemicals use through licensing and monitoring processes.

\section{Where is innovation needed to promote synergies?}

- Better understanding of how fisheries and renewables can work alongside one another, with innovation not limited to technological solutions but also tackling the governance challenges to coexistence.

- Seafood needs to be incorporated into mainstream food policies instead of siloed and addressed under marine or agriculture or environment (for example, in DEFRA).

- Value needs to be captured more locally in production.

- Innovation may be constrained when profit is low; sharing opportunities and understanding can support progress.

- We need to benchmark standards and learn from best practices, including the private sector, especially for areas such as precision catching, adding value, and market knowledge.

- There is a need for a simpler, clearer approach to management and monitoring in aquaculture.

- How to influence behaviour change - including particular focus on consumer behaviour, linked to steering market / advertising companies to act in a way that supports sustainable choices and fishing practices.

- In terms of governance, the role of Regional Inshore Fisheries Groups (RIFGs) in delivering sustainable management of fisheries could be further examined, considering new statutory powers and legislation to modernise fisheries and their management. This should include integration with marine planning systems.

What type of indicators, data and evidence are needed to measure progress towards sustainable seafood production and consumption?

- Transparent and traceable data management systems are needed through supply and value chains, that facilitate comprehensive, independent and integrated assessments of all aspects sustainability, including the climate impact of seafood.

- Indicators are needed which enable measuring progress at different scales, including local, regional and national, across the multiple sectors within seafood.

- Economic performance at local and national level is pertinent to understanding change in the sector.

- Public feedback on progress is also valuable when considering impacts and relevance to society.

- The use of digital technology / electronic monitoring throughout the Scottish fleet and onboard vessels operating within Scottish waters is important in contributing evidence for measuring progress. 
What research and knowledge can help us manage synergies and trade-offs in nature-based solutions?

True interdisciplinarity is needed, with the inclusion of social research and focus on governance innovations recognised as increasingly important to unlock potentially positive outcomes. This should be accompanied by further moves to a common 'currency' or language for science-policy discussions (e.g. 'natural capital', 'nature-based solutions', 'ecosystem services'), working across natural, social and economic components. Whichever terms are used, it is key to understand the different ecosystem functions and services and associated pressures to inform decision-making. Supporting science is important to inform management interventions, whether as protection, restoration, recovery or enhancement of ecosystem functions.

The following knowledge areas were highlighted:

- New methods to evaluate, value and understand changes in the cultural/social ecosystem services that flow from nature, including amenity, access, health and well-being.

- Clarify the opportunities and priorities of nature-based solutions, giving attention to climate adaptation as well as mitigation and wider benefits from nature, while also being clear on the respective timescales on which nature-based solutions can be delivered (compared to the often more immediate needs that must be met by technology and behaviour change).

- Comparing pressures of human activities such as fishing against the distribution of blue carbon sediment 'hotspots'.

- In addition to blue carbon benefits, it is important to understand the benefits of such ecosystems/habitats and to address the multiple pressures that these areas are facing.

Where is innovation needed to promote synergies?

- Economic/financial tools are needed to leverage private and public investment ('blue finance') in nature to tackle externalities, from both central (national) and local sources, including partnering of public and private organisations.

- Public engagement and educational innovation for ocean literacy, including engagement techniques that build trust between stakeholders / experts / youth and decision-makers.

- Science and innovation for safe and effective habitat and species restoration.

- Coastal areas provide a concentration of potential for nature-based solutions, but also a concentration of human interaction with the marine environment, so could benefit from innovative governance for a programme of strategic and technical support.

What type of indicators, data and evidence are needed to measure progress?

- There is a confusing array of existing indicators across national, regional and international biodiversity reporting obligations, often with some uncertainty of data quality and granularity. Clarity on these will be required, including any further known gaps in our knowledge.

- Measures of social acceptability and related variables are needed, with an understanding of how these vary geographically / culturally.

- Socio-economic analyses with qualitative evidence, avoiding looking only at numbers.

- Some features of nature and associated benefits can recover quickly, others are slow, but potentially very substantial and important. A mix of rapid analysis and long-term surveillance is necessary, and a balance of broadscale and detailed site-specific monitoring. 
- For perspective, we must put data and evidence in the context of prior conditions. However, we must accept that our understanding of baselines are not always informed by adequate data, but this does not invalidate what is known from informal records and local knowledge.

- Is no loss of biodiversity a reasonable goal/the right approach when biodiversity will change as a result of climate change? Should we re-frame our progress and National Performance Framework indicators for biodiversity to reflect the structure and function of biological communities and ensure ecosystem resilience?

\section{Theme 4. Circular Economy}

What research and knowledge can help us manage synergies and trade-offs for a circular economy?

- In addition to new research, there is a need use existing data in a better way, including through visual representation to help identify connections and trade-offs in the system.

- How best to organise, store and make available data on the full life cycle and fate of materials, resources and any associated waste, then supporting knowledge on where the resource inefficiencies and business opportunities are.

- Research on (and investment for) infrastructure solutions that can be resilient to climate change (e.g. investment in underwater motorways in Norway, which brought together engineering, marine science, social and population dynamics).

Where is innovation needed to promote synergies?

- Innovation is about new ways of thinking and doing, not just technology. This includes having the right people involved, which must include targeted end-users. Innovation in governance approaches, data flow and business models could achieve a great deal relatively quickly.

- More advanced cumulative, full-system and life cycle assessments of commercial activities are needed to portray a holistic view of the consequences of our policy actions under different scenarios, for example to understand the broader implications in the whole system of actions/targets for wind energy development.

- Data systems to support full transparency and traceability of resources through supply chains, including the fate of any residual waste, also supporting advances in environmental certification standards. By revealing inefficiencies businesses can be expected to respond to the financial incentive and CSR-imperative to close the materials loop as far as possible.

- Development of new feed sources for finfish aquaculture, such as from plant-based sources or insect-protein bred on food waste.

- Alternative uses for waste, including dredge material, biofouling and aquaculture waste.

What type of indicators, data and evidence are needed to measure progress?

- We need a long-term perspective and move beyond the monetary focus/economic growth indicators to support a circular economy.

- Life Cycle Analysis and real-time tracing of resource use and waste is essential in monitoring progress in a circular economy, tailored to different sectors.

- There is a need to consider the circular economy in rural coastal communities as well as national level, seeking and measuring benefits for small and medium enterprises that operate locally for energy and resource efficiency across whole sectors. 